\title{
Nouvelles Frontières Pédagogiques: Entre Précarité Alimentaire et Gaspillage
}

\author{
Sara Bornatici \\ Università Cattolica del Sacro Cuore, Alta Scuola per l'Ambiente, Brescia, Italie
}

\begin{abstract}
Penser et construire un espace et un temps de prospérité et de bien-être pour tous les habitants de la planète est un défi moral à relever pour donner naissance à des communautés innovantes et inclusives qui se reconnaissent réciproquement.

L'analyse d'un certain nombre de périphéries existentielles permet de considérer ces dernières comme de précieuses sources, une stimulation pour provoquer les consciences vers un engagement éthique qui valorise et encourage la responsabilité individuelle et collective. Promouvoir des processus éducatifs durables qui induisent chacun à se comporter en imaginant que ses propres choix pourraient être suivis par toutes les personnes qui peuplent notre planète signifie voir l'humain à la fois comme valeur et lien, point de départ et d'aboutissement, ressource pour générer un projet partagé qui prenne en compte et renforce des processus de participation et de réflexion inédits.

Cet essai aborde du point de vue pédagogiqueéducatif le thème de la pauvreté alimentaire et affronte les significations culturelles et sociales d'un phénomène, celui du gaspillage, pour comprendre si et de quelle façon ce dernier pourrait être problématisé et, par la suite, lu comme une ressource du point de vue pédagogique en posant au centre de l'intérêt scientifique le développement humain intégral et la prise de responsabilité de chacun pour la construction de la société de demain.
\end{abstract}

\section{Introduction}

Dans la société occidentale contemporaine, caractérisée par une tension accentuée vers le consumérisme et la frénésie des rythmes de vie, on assiste de plus en plus intensément à une forte augmentation des inégalités socio-économiques et à des processus d'appauvrissement qui touchent les couches les plus faibles de la population. Quoique disposant d'un progrès technique et matériel sans précédent, une part importante de l'Humanité vit dans la pauvreté, phénomène qui constitue un frein au développement et un accélérateur des crises environnementales.
Mais la pauvreté, c'est aussi le non accès à des ressources intangibles comme l'éducation. "Parmi les aspects multidimensionnels de la pauvreté, un élément stratégique est celui de l'accès à l'éducation et des interrelations qui opèrent au sein des politiques publiques (globales et sectorielles). Dans le cadre de la lutte contre la pauvreté, l'éducation doit se développer malgré la pauvreté, pour permettre de sortir de la pauvreté» [1].

Dans le contexte actuel, la formation des ressources humaines s'avère être un aspect performant pour l'avenir de notre civilisation.

Éduquer à la pauvreté pour édifier le bien commun, tel est le défi thématique que traite cet essai en considérant le projet éducatif sous le signe de l'apprentissage permanent (lifelong learning).

L'analyse présentée dans les pages suivantes part d'une réflexion sur la pauvreté alimentaire et l'utilise comme instrument pour diffuser au sein des établissements scolaires une culture du développement durable, entendue comme forme de capital social indiquant le degré de cohésion civique et la profondeur des rapports de solidarité [2].

\section{Questions émergentes}

Une vue d'ensemble de l'état de l'alimentation dans le monde montre qu'il existe un fossé considérable dans la population mondiale entre ceux qui n'ont pas suffisamment de quoi se nourrir pour vivre et qui souffrent de faim et de dénutrition et des situations d'excès entraînant le gaspillage de ressources. L'étude des transformations qui touchent l'ensemble de la planète fait état d'une hausse de l'inégalité entre pays riches et pays pauvres, et le gaspillage se configure comme le paradoxe de la pénurie dans l'abondance : peu ont trop et beaucoup ont peu. Dans un monde de sept milliards de personnes, dont le nombre est destiné à croître jusqu'à atteindre neuf milliards d'ici 2050, gaspiller la nourriture est un véritable outrage économique, environnemental et éthique ; et pourtant, selon les données fournies par Coldiretti, en 2017 près de 2,7 millions de personnes en Italie ont bénéficié des aides alimentaires en ayant accès aux soupes populaires ou aux colis alimentaires qui répondent 
davantage aux attentes des nouveaux pauvres (retraités, chômeurs, familles avec enfants) qui, par honte, préfèrent cette forme d'aide plutôt que la consommation de repas gratuits dans les structures caritatives. Parmi les plus démunis, on compte 455 000 enfants de moins de 15 ans, près de 200000 personnes âgées de plus de 65 ans et près de 100000 sans domicile fixe [3].

$\mathrm{Au}$ niveau mondial, la FAO signale comment « Aujourd'hui 815 millions de personnes souffrent de la faim, mais au-delà du manque de calories et de la maigreur, la malnutrition se caractérise aussi par des carences en micronutriments, par le surpoids et l'obésité. Il reste beaucoup à faire pour mettre fin à la malnutrition et accéder à un monde libéré de la faim. Alors pourquoi ne pas adopter une approche amusante et efficace pour éduquer les enfants, et du même coup atteindre aussi les adultes ? ».

Cette contribution entend proposer une réflexion sur la nourriture comme génératrice de bonnes pratiques qui promeuvent l'humanum et en enrichissent l'identité. Reconnaître à la nourriture ses valeurs salutistes, culturelles et affectives revient à véhiculer la transmission des savoirs entre les générations et cultiver des valeurs d'affectivité, de convivialité, de connaissance réciproque. La conscience que c'est dans l'enfance que s'établissent et se consolident les habitudes alimentaires de chacun impose à l'école un rôle déterminant dans la promotion de modes de vie corrects. Plus précisément, le moment du repas peut représenter pour les élèves une importante occasion d'éducation alimentaire, de socialité et de partage et être lié à des expériences de don, en réseau avec les structures caritatives présentes sur le territoire.

Ainsi considère-t-on la pauvreté, comme l'indique Vico, non comme « un ennemi à vaincre mais comme un oxymore à saisir et à interpréter dans ses significations les plus profondes et dans l'opportunité qu'il puisse en ressortir une vérité riche de sens» [4].

\section{Littérature de référence}

L'OMS, Organisation mondiale de la santé, dans le document Malnutrition et situations d'urgence (disponible en ligne http://www.who.int/features/qa/malnutritionemergencies/fr/), souligne la manière dont la malnutrition prend aujourd'hui de nombreuses formes en incluant :

La dénutrition, phénomène qui a lieu lorsque la personne n'a pas assez à manger et souffre par conséquent de pathologies liées à la mauvaise alimentation telles que la malnutrition chronique ou le paludisme. Une alimentation non équilibrée pendant les mille premiers jours de vie peut avoir comme conséquence un retard de croissance avec des effets irréversibles à long terme sur le développement physique et mental du jeune enfant. Les données de l'OMS estiment à 156 millions les enfants qui en 2015 ont souffert d'un retard de croissance.

Les carences en micronutriments, lorsque l'alimentation n'apporte pas suffisamment de vitamines et de minéraux importants ;

Le surpoids et l'obésité, qui sont liés à une mauvaise alimentation ou à une alimentation déséquilibrée résultant en une surconsommation de calories, et qui sont souvent associées à la sédentarité. Ils peuvent entraîner des maladies non transmissibles telles que les cardiopathies, l'hypertension, le diabète.

La société occidentale est surtout affligée par la globésité,

(http://www.who.int/nutrition/topics/obesity/en/) la dernière de ces trois typologies de malnutrition : des données alarmantes signalent qu'un enfant sur trois en Italie est en surpoids et présente une série de graves problèmes de type sanitaire, aussi bien physiques que psychologiques, destinés à l'accompagner, en s'aggravant, à l'âge adulte aussi.

Compte tenu de ces évidences, une prévention soignée et précise sur le plan médical s'avère nécessaire mais ne peut toutefois être séparée d'une action éducative systématique dès le premier âge de la vie. Pati [5] remarque que « tout au long de la première enfance, il y a la nécessité de favoriser l'enfant dans la prise de bonnes habitudes. Celles-ci lui servent pour ordonner son existence dans le monde des choses et des hommes en lui permettant de leur attribuer des significations précises ».

En accomplissant cette tâche, famille et école prennent des fonctions différentes mais complémentaires : "si à la maison le rapport avec la nourriture se construit davantage à travers des formes éducatives inconscientes, qui partent de l'émotivité, d'habitudes et de routine, l'école peut créer de nouveaux parcours, de nouvelles connexions entre aspects émotifs et cognitifs en valorisant et en restructurant, si nécessaire, l'expérience personnelle et familiale »[6]. Elle est donc appelée à éduquer enfants et familles à la conscience de l'importance d'une alimentation saine en vue de leur bien-être psychophysique et de leur qualité de vie en soulignant qu'alimentation n'équivaut pas seulement à nutrition, acte nécessaire à satisfaire un besoin physiologique, mais à identité, racines, culture ; en partageant le repas, les enfants à l'école se mettent en relation et dialoguent, se découvrent eux-mêmes, leurs goûts et en même temps s'approchent de l'autre.

Aujourd'hui plus qu'hier, l'école représente le principal lieu de rencontre entre pairs ; elle est un lieu crucial de socialisation pour faire des expériences de croissance communautaire. Une attention renouvelée pour l'enfance, une conception éducative durable qui identifie des itinéraires 
formatifs dédiés, doit partir du postulat que la dimension sociale est étroitement liée à l'éducation puisqu'elle exprime la nature relationnelle de la personne [7].

Il est évident que dans les horizons culturels actuels, la nourriture joue un rôle emblématique et se configure comme ressource incontournable pour réaliser une façon de faire école qui soutienne l'activité exploratrice de l'enfant : être ensemble à table va au-delà d'un simple geste de routine et prend des significations importantes pour réfléchir et agir de façon située. Dans ce scénario, la réflexion pédagogique est appelée, à travers une interprétation critique, à élaborer de nouveaux réseaux de connaissance et d'action dans la perspective du bien commun et de la durabilité. Éducation, recherche et formation se configurent comme instruments significatifs pour garantir l'équité et la reconnaissance $\mathrm{du}$ droit fondamental à une alimentation saine, sûre et suffisante pour l'humanité toute entière.

Emblématiques sont les lignes directrices pour l'éducation alimentaire promulguées par le MIUR, le Ministère de l'Éducation, de l'Université et de la Recherche italien (2015) [8]; à la lumière des héritages éducatifs et culturels d'EXPO 2015, le document identifie quatre pistes de travail fondamentales, chacune étant significative par rapport à un aspect particulier de la relation avec la nourriture, qui peuvent aider enseignants et éducateurs à produire de la conscience chez l'enfant en partant du niveau individuel (moi, la nourriture dans mon assiette et mon corps), pour arriver à travers la réalité spatio-temporelle proche (moi, la nourriture dans ma journée, l'école et la famille) à celle plus lointaine (moi, la nourriture, la saison, les amis et le territoire où je vis) et à la dimension interculturelle et planétaire (moi, la nourriture et la société dans le monde et dans le temps).

\section{Débat. Le paradoxe du gaspillage alimentaire}

Parmi les nombreuses implications articulées et complexes que la pauvreté porte en soi, il convient d'isoler un phénomène qui semble s'opposer à la pauvreté elle-même, s'écartant nettement de ce qui vient d'être décrit : en Italie, 8,8 millions de tonnes de nourriture (www.fao.org) sont perdues et gaspillées chaque année ; ces quantités pourraient satisfaire le besoin alimentaire des trois quarts de la population de notre pays.

En littérature, on a pour habitude de distinguer entre food losses, à savoir les pertes alimentaires qui ont lieu en amont de la filière agroalimentaire, essentiellement durant l'ensemencement, la culture, la récolte, le traitement, la conservation et la première transformation agricole, et food waste, à savoir les gaspillages qui ont lieu durant la transformation industrielle, la distribution et la consommation finale (Barilla Center for Food and Nutrition).

Le Waste Resources Action Program (WRAP, http://www.wrap. org.uk) subdivise le concept de food waste en trois catégories :

- gaspillage évitable (nourriture et boissons qui, avant d'être jetés à la poubelle, sont encore mangeables, tels que tranches de pain, pommes, viande, etc.) ;

- gaspillage potentiellement évitable (nourriture et boissons qui peuvent être consommés, tels que la peau de certains fruits, la croûte des fromages, et également la nourriture qui peut être récupérée si elle est cuisinée, par exemple les épluchures de pomme de terre) ;

- gaspillage inévitable (os, coquilles d'œufs, pelures d'ananas, etc.). [9]

Concernant les deux premières catégories, les données présentées par l'Observatoire de Waste Watcher (http:// www.sprecozero.it/waste-watcher/) sont intéressantes car elles révèlent que le gaspillage annuel de nourriture en Italie s'élève à près de 16 milliards d'euros, soit $1 \%$ du PIB. On gaspille surtout dans les ménages : près de $145 \mathrm{~kg}$ de nourriture par an et par famille, soit $75 \%$ du gaspillage global en Italie, correspondant à un coût annuel de 360 euros.

Il est manifeste que ces définitions quantitatives du phénomène en question sous-tendent d'importants éléments en termes de qualité et de valeurs qui, du point de vue d'une réflexion pédagogique, permettent de dégager de manière distinctive les dimensions éthique et éducative que soulève la thématique en question. Nous nous trouvons en effet face à une véritable urgence sociale qui présente de lourdes répercussions sur le plan environnemental, économique, éthique : en ce qui concerne l'impact sur l'environnement, on peut considérer, à titre d'exemple, la quantité d'eau ou l'extension de sol agricole utilisées pour produire la nourriture gaspillée ; sur le plan économique, il suffit de rappeler que la valeur du gaspillage alimentaire au niveau mondial avoisine les 1000 milliards de dollars par an ; dernier point, mais non des moindres, il convient de considérer les implications emblématiques du point de vie social et éthique : le gaspillage de nourriture souligne une contradiction intolérable face au nombre croissant de personnes dans le besoin qui chaque jour font appel à des organisations caritatives pour avoir un repas chaud. La carence, l'excès et le gaspillage sont des paradoxes qui renvoient au même problème et nécessitent de bonnes pratiques pour être corrigés.

La lutte au gaspillage alimentaire et à la culture des déchets est donc une première réponse pour agir de 
façon concrète en protégeant et en améliorant la qualité de vie globale.

Cet énorme challenge a été affronté au niveau mondial, notamment dans le cadre de l'Expo 2015, manifestation universelle qui a demandé à tous les acteurs publics et privés et à la société civile de s'engager concrètement pour créer un environnement favorable fondé sur l'équité, sur le respect et sur le caractère sacré de la vie. Le concept de gaspillage fait référence à une utilisation non correcte ou non profitable, voire même à une non-utilisation, d'une ressource. Sur le plan du développement durable, ceci implique une consommation doublement inutile, d'abord pour la production et ensuite pour l'écoulement du produit inutilisé. Si, d'un côté, ceci souligne la nécessité d'une meilleure gestion et d'une meilleure distribution des ressources alimentaires au niveau mondial, national et local, il est évident, de l'autre, qu'il n'est plus possible de repousser davantage encore un travail qui, dans une approche ascendante, engage chaque citoyen à mener une réflexion et une action dans la certitude qu' " une réciprocité solidaire de devoirs associe les destins humains » [10]. Le thème du gaspillage peut par conséquent être considéré comme un problème réellement éducatif et être donc étudié d'un point de vue pédagogique aussi. Dans une logique d'échange et de négociation des connaissances, la culture d'un humanisme planétaire impose une révision globale de nos modes de vie, un engagement renouvelé de tous et de chacun afin que nous puissions être les promoteurs d'une culture de paix qui nous permette de retrouver avec compétence les valeurs de la fraternité et du bien commun. Récupérer la dimension de la sobriété est un engagement qui s'adresse à notre quotidien d'aujourd'hui mais aussi aux générations futures dont l'existence est liée à nos choix alimentaires actuels.

Il est nécessaire de tenir compte de nouvelles sensibilités et de comportements davantage responsables capables de faire prévaloir la valeur de la personne sur d'autres logiques liées au profit. Face à l'indigence et à la pauvreté, il n'est pas possible de choisir l'indifférence, de maintenir les autres à distance de façon à éviter toute implication, comme pour être épargnés de toute forme d'investissement émotif vis-à-vis de l'autre.

Pour préserver les ressources naturelles et apprendre dans la société de la connaissance, en innovant des processus organisationnels et des politiques institutionnelles la réflexion pédagogique doit générer une disponibilité cognitive et émotive à sortir de soi pour s'ouvrir à l'autre et est appelée à proposer de nouveaux horizons de pensée et d'action en entrant dans le concret, dans le sens et le vécu, dans les processus constitutifs, dans les pratiques.

Compte tenu de ces indications, la cantine scolaire devient un lieu d'action mais en même temps de sens et de pensée et a besoin d'une conception pédagogique attentive aux petits gestes quotidiens.

Un repas responsable peut se configurer comme espace de création et de partage de la culture en entamant des confrontations conceptuelles significatives vouées à une amélioration de l'existant. Ceci pousse la réflexion pédagogique « à repenser la 'densité ontologique' de la conceptualité formative en référence aux valeurs éducatives » [11] pour dire la durabilité de la vie.

Inclure aussi dans la didactique le temps de la cantine signifie opérer pour la construction d'une école où les bonnes pratiques parviennent à être l'horizon de nouveaux scénarios de sens en promouvant des connaissances et des compétences en mesure de transformer et d'engager des parcours de croissance emblématiques qui non seulement auraient le caractère de la fonctionnalité mais qui s'exprimeraient dans la capacité expressive. Le futur d'une école plurielle consiste à garantir de vastes niveaux de communication autour de significations et de valeurs partagées, à attribuer au soin et à la relation les traits les plus qualifiants de l'humain.

Il s'agit de réaliser des interventions conceptuelles actives qui encouragent à la pensée, à la re-conception et à l'action « en acceptant le risque et l'aventure de l'exposition à ce qui vient de dehors, à l'événement de l'autre, à faire l'expérience de l'autre et des autres $\gg$ [12].

Associer la nourriture aux valeurs d'affectivité, de la convivialité, de la connaissance réciproque, de la découverte des diversités peut advenir par exemple à partir d'une lecture de la grande quantité de nourriture gaspillée à la cantine et des solutions que les enfants eux-mêmes, guidés par leurs enseignants, peuvent proposer pour favoriser la croissance liée à des dimensions civiques telles que le sens de l'épargne, le respect des ressources et le soutien aux catégories les plus faibles.

Dans un tel contexte, nombreuses sont les initiatives qui encouragent et facilitent dans le cadre des cantines scolaires le recyclage de la nourriture et des produits alimentaires encore parfaitement comestibles à des fins de solidarité sociale, en promouvant la responsabilisation des personnes engagées à travers des actions et des réflexions en mesure de lutter contre le gaspillage alimentaire.

Le gaspillage alimentaire, pratique répandue dans de nombreux contextes occidentaux à l'instar d'autres formes de mauvaise éducation dans le rapport avec la nourriture, entraîne non seulement un préjudice économique mais aussi une forme de grave injustice sociale vis-à-vis des pauvretés émergentes.

Bien que les données du Food Sustainable Index mentionnent l'Italie parmi les Pays qui sont en train de faire davantage pour lutter contre le gaspillage de la nourriture, comme le prouvent également les indications offertes par la récente loi Gadda 166/2016 166 portant « Dispositions relatives au don 
et à la distribution de produits alimentaires et pharmaceutiques à des fins de solidarité en vue de la limitation du gaspillage », les données de chaque école ne sont pas encourageantes.

\section{Une bonne pratique: le project Think, eat, don't waste.}

Sans prétention d'exhaustivité et à titre purement d'exemple, il est utile de réfléchir sur une donnée significative issue de la recherche Think, eat, don't waste. Previeni lo spreco alimentare, educa! menée par la Haute École pour les Études environnementales (Alta Scuola per l'Ambiente) de l'Université Catholique du Sacré-Cœur durant l'année académique 2016/2017 en collaboration avec « Banco Alimentare » (Banque Alimentaire d'Italie) dans douze écoles primaires de la province de Brescia.

Le pesage des déchets, effectué pendant une semaine à la fin du repas à l'école, a relevé que chaque enfant gaspille en moyenne 100 grammes de nourriture.

Dans une perspective éducative, cette opération a permis de tracer quelques directions de sens et des pistes opérationnelles qui ont permis aux enfants de réfléchir et d'avoir une approche consciente à l'alimentation.

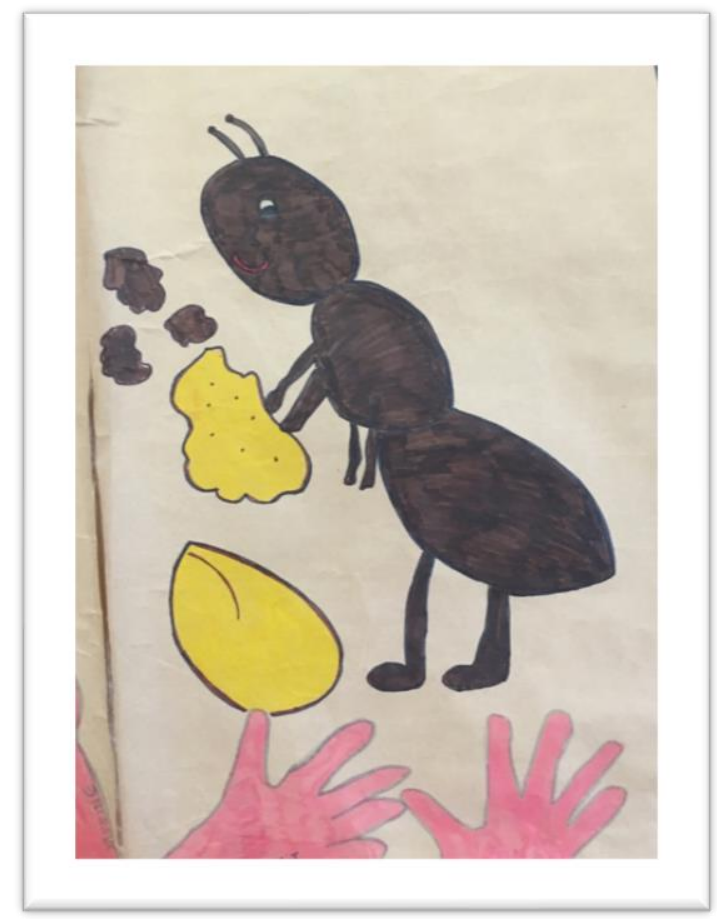

Figure 1. Le travail d'une classe sur le thème du gaspillage

En premier lieu, il a été possible, à travers des conversations et des groupes de discussion, de rechercher avec les enfants les raisons d'un tel gaspillage ; les causes sont multiples et ne sont pas toutes attribuables aux caractéristiques organoleptiques des aliments proposés mais surtout à l'absence de valeur attribuée à l'alimentation par les enfants et une dévalorisation diffuse des propositions alimentaires au sein du noyau familial.

Dans ce contexte, affirme Isidori [13], il s'avère fondamental de procéder à « la conscientisation des personnes vis-à-vis de l'alimentation elle-même, à leur responsabilisation vers une conscience développée selon une perspective pédagogique et formative qui n'est pas dépositaire mais « active », " dynamique » et surtout " critique ». L'alimentation peut donc être considérée comme un langage qui permet de gérer son propre univers de symboles et de significations, une modalité d'expression de sa personne, de son existence dans le monde.

En second lieu, l'opération de pesage des déchets a fait réfléchir sur un phénomène, à savoir le gaspillage alimentaire, qui pose des interrogatifs sur les déséquilibres de consommation dans le monde et sur la disparité sociale entre ceux qui gaspillent et ceux qui n'ont pas de quoi manger. Le gaspillage de nourriture résonne donc comme un appel étique et nous rappelle que « nous devons nous engager à rendre à la terre et, par voie de conséquence, à nos enfants et aux générations futures cette énergie de la vie que nous empruntons tous les jours et que nous ne devons plus consommer en courant le risque de la détruire » [14].

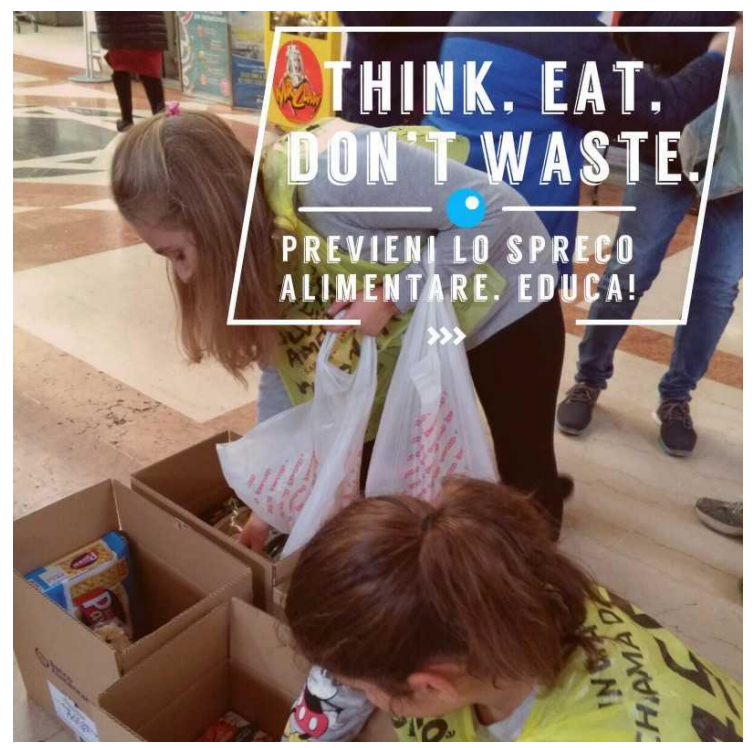

Figure 2. Les élèves participent à une initiative de solidarité pour recueillir de la nourriture pour les personnes dans le besoin

Augmenter chez les enfants la conscience de la réalité du gaspillage et des pauvretés alimentaires signifie les faire entrer en contact avec des thèmes qui concourent à la formation d'un développement humain intégral et qui, dans le domaine pédagogique, 
s'enrichissent de nouvelles interprétations en en valorisant la nature éducative et formative. «Une éducation du futur citoyen à l'école ouvre la voie à la participation des élèves à propos de multiples aspects de la vie scolaire» [15] et à la connaissance des valeurs.

\section{Conclusions}

Le moment du repas à l'école doit bien entendu et se configurer comme une opportunité et une occasion pour promouvoir une éducation alimentaire agie et située, non traitée de façon réductrice comme interruption des activités scolaires. L'école peut jouer au moment du repas aussi un rôle inédit de production culturelle et se proposer comme un espace d'éducation à de nouveaux styles de vie plus responsables et solidaires. Assumer la responsabilité de l'Éducation Alimentaire tout au long du parcours scolaire signifie porter attention aux dimensions de la culture, de l'histoire, de la valeur de la nourriture ; cela implique également le souci des relations humaines, la recherche de conditions durables de bien-être et pousse à repenser à ses propres racines et à l'identité de chacun ; ouvre, entre équité et durabilité, une réflexion sur les aspects valoriels de la nourriture qui gagne donc une position comme interlocutrice privilégiée de thématiques environnementales, économiques et sociales.

La FAO à travers Save the food, initiative mondiale de réduction des pertes et du gaspillage alimentaires, signale quatre piliers sur lesquels fonder un parcours authentique de promotion de la valeur de la nourriture qui inclut en première position la sensibilisation pour permettre une meilleure connaissance du problème et favoriser ainsi la modification des comportements des consommateurs.

L'identité individuelle, même dans le monde de l'enfance, semble de plus en plus trouver un ancrage dans la reconnaissance sociale dérivant de la possession des biens, dans le fait de s'aligner à un style de vie conformiste, homologué et peu original. La réflexion pédagogique relève le défi consistant à « accompagner et soutenir le développement de l'identité à travers une succession incessante de différenciations et d'intégrations » [16].

Guider les enfants dans le processus d'exploration émotive et culturelle de la nourriture signifie reconnaître au thème du gaspillage une valeur écologique et sociale qui appelle en cause dans les débats sur l'environnement le thème de la justice « pour écouter le cri de la terre et le cri des pauvres » [17].

Un aspect qui peut être développé et décliné à partir d'une réflexion sur le gaspillage est donc lié à la « logique du don, qui sait offrir sans rien attendre en retour, seulement parce qu'on veut instaurer une relation qui - on le devine - peut s'avérer vraiment humaine si elle est marquée par la gratuité » [18]

Outre la sensibilisation, ce qu'il ressort du document de la FAO, c'est la nécessité de développer un esprit de collaboration et de coordination d'initiatives mondiales en favorisant des partenariats permettant de développer, planifier et répartir les ressources de manière optimale, le développement de politiques, stratégies et programmes qui conjuguent une approche à la filière alimentaire pour l'évaluation des pertes avec l'analyse des coûts et des profits afin de déterminer quelles interventions seraient les plus efficaces et le soutien à des programmes d'investissement et aux projets.

Il s'agit donc, à travers l'éducation alimentaire, d'interpréter et de mettre en œuvre un projet de construction du bien commun dans le signe de l'apprentissage continu, dans la conscience qu' " une société qui ne se soucie pas de ses jeunes générations et de leurs lieux de vie électifs se désintéresse de l'investissement primordial, à savoir celui sur le capital humain » [19].

Le but qu'il faut réaliser c'est que chacun puisse parvenir «à s'inventer lui-même, par lui-même, et à créer sa place dans la société en la faisant réciproquement évoluer elle-même. C'est là le succès de l'éducation, l'autonomie de chacun dans une école qui a pour but la réussite de tous ses élèves» [20].

\section{References}

[1] N. Henaff, M. Lange and J. Martin, "Revisiter les relations entre pauvreté et éducation.", Revue Française de Socio-Économie, Paris, 3, (1), 2009, pp.188.

[2] P. Malavasi, "Pedagogia dell'ambiente. Tra degrado, sostenibilità e sviluppo umano integrale", Pedagogia Oggi, (2), 2013, p. 209.

[3] Coldiretti 'Consumi. 2,7 mln di italiani costretti alla mensa dei poveri'; https://www.coldiretti.it/economia/datie-numeri-poverta-in-italia [Accessed: 19-October-2018].

[4] Vico, G. (2007) 'Bambini di strada e 'nuovi poveri' interpellano l'educazione'. In Id (Ed.), Orientamenti per educare alla cittadinanza, Vita e Pensiero Milano, p.48.

[5] Pati, L., Livelli di crescita. Per una pedagogia dello sviluppo umano, La Scuola, Brescia, 2016, p.49.

[6] Birbes, C., Nutrirsi di relazione. Una riflessione pedagogica tra cibo e educazione, Pensa Multimedia, Lecce-Brescia 2012, p.110.

[7] Pati, L., Pedagogia sociale. Temi e problemi, La Scuola, Brescia, 2007, p.49.

[8] MIUR, (2015). 'Linee guida MIUR 2015 per l'educazione alimentare', Available from http://www.istruzione.it/allegati/2015/MIUR_Linee_Guida 
_per_l'Educazione_Alimentare_2015.pdf [Accessed: 19October-2018].

[9] Wrap, (2018). 'Food waste reduction', Available from http://www.wrap.org.uk/food-waste-reduction [Accessed: 19-October-2018].

[10] Devaud, E., L'école affirmatrice de vie, Librairie de l'Université, Fribourg, 1938, p.27.

[11] Vischi, A., Temi e prospettive dell'Alta Formazione. Tra ricerca pedagogica e responsabilità intergenerazionale, Pensa Multimedia, Lecce. Brescia, 2012, p.149.

[12] Laneve, C., "Verso un nuovo paradigma di insegnamento nell'era digitale", In Rivoltella, P.C., Felisatti, E., Di Nubila, R., D., Notti, A.,M., Margiotta, U., (Eds), Saperi pedagogici e pratiche formative. Traiettorie tecnologiche e didattiche dell'innovazione. Saggi in onore di Luciano Galliani), Pensa Multimedia, Lecce_Brescia, 2016, p.122.

[13] E., Isidori, "La pedagogia dell'alimentazione: contributo ad una teoresi educativa". Formazione, lavoro, persona, Rivista Online, 14, 2015, p.125.

[14] Mattarella, S., Intervento del Presidente della Repubblica S. Mattarella all'Expo 2015. Retrieved 5, august,2018,

from http://www.quirinale.it/elementi/1045,2015, [Accessed: 17-October-2018]

[15] Haeberli, P., Pagoni, M., "Introduction générale:Collaborer c'est un ordre? La partecipation à l'école en trois questions". In P. Haeberli, M. PagoniI, O. Maulini. La partecipation des élevés: effect de mode ou nécessité?, L'Harmattan, Paris, 2017, p. 16.

[16] Dozza, L., Educazione permanente nelle prime età della vita. In L. Dozza, S. Ulivieri (Eds.), L'educazione permanente a partire dalle prime età della vita, Franco Angeli, Milano, 2016, p.65

[17] Francesco, Lettera Enciclica Laudato sì. Enciclica sulla cura della casa comune, 2015, n.49.

[18] A. Bellingreri, "Il mangiare e il bere come figure rivelative dell'esperienza umana", Formazione, lavoro, persona, 14, 2015, p.42.

[19] Malavasi, P., Expo education Milano 2015. La città fertile, Vita e Pensiero, Milano, 2013, p.76.

[20] Labelle, J.M., Apprendre les uns des autres. La réciprocité source d'éducation mutuelle, L'Harmattan, Paris, 2017, p. 120. 